Using one example of a completed EIT-Health funded implementation project, JIGSAW-E (Joint Implementation of Guidelines for oSteoArthritis in Western Europe), this presentation will highlight some of the challenges of implementation such as: understanding the context; competing priorities; lack of time and funding; stability of the workforce; and differing health care systems. It will describe the use of Knowledge Mobilization and Communities of Practice as methods for overcoming these challenges and optimizing holistic treatments.

The presentation will offer some of the key factors for implementation such as 'leading from the middle' and brokering across silos; working with patients and the public 'Patients are the most under-used resource in any health care system'; using theories, models and frameworks to plan, understand and evaluate implementation; using pilot sites to build momentum with dedicated Communities of Practice; and agreeing what success should look like.

Disclosure of Interests: None declared

DOI: 10.1136/annrheumdis-2019-eular.8464

\section{SP0123 NEW TREATMENTS: WHY SHOULD WE WAIT?}

Daniel Morales. University of Dundee, Population Health and Genomics, Dundee, United Kingdom

Background: Improving access to new and established medicines for rheumatological disease is of public health importance. However, drug development processes, medicines regulation and health technology requirements may significantly impact on when a new medicine become available to be used by health care professionals.

Objectives: The objectives of this session are to present some of the challenges and opportunities for accessing new and established medicines for rheumatological disease.

Methods: Drug development processes, medicines regulation and health technology requirements may significantly impact on when a new medicine become available to be used by health care professionals. Although these requirements may be perceived as barriers, many of these requirements are in place to ensure a medicines benefit risk profile is favorable and is cost effective in terms of quality of life. This session will briefly discuss issues around the process of making medicines newly available from drug development to gaining new market approvals with an emphasis on rheumatological disease, including the post-authorization safety requirements that are in place to safe guard public health.

Disclosure of Interests: None declared

DOI: 10.1136/annrheumdis-2019-eular.8542

FRIDAY, 14 JUNE 2019

13:30:00 - 15:00:00

\section{New assessments in clinical practice}

\section{SP0124 WHICH MEASURES TO USE TO ASSESS REMISSION IN RHEUMATOID ARTHRITIS}

Annamaria lagnocco. Academic Rheumatology Unit Universita degli Studi di Torino, Academic Rheumatology Centre, Turin, Italy

Remission is the ideal target of treatment in rheumatoid arthritis (RA) patients. However, the definition and characterization of remission is still a theme of discussion and its assessment can be a challenging issue in RA clinical practice. Indeed, nonetheless the new treatments and therapeutic strategies for RA make remission possible, sub-clinical inflammation can still be present both at the joint site and at the patient level, leading to damage progression and to occurrence of flares.

Clinically, remission entails persistent absence of any sign of joint and systemic inflammation. However, the presence of some residual joint inflammatory activity can be accepted by current clinical indexes such as DAS28, SDAI, CDAI and Boolean remission, although the latter is clearly based on more stringent parameters $(1,2)$. Based on those criteria, and particularly with the use of SDAI and Boolean cut-offs, complete and persistent remission is then quite uncommon in late RA, while it seems to be more easily possible in early disease (3). Another interesting issue to consider in clinical practice is the presence of sustained remission which seems to occur less frequently than remission at different point times and with only a minority of cases reaching a complete disease recovery (4).

Depending on the modality that is used, the assessment of remission by imaging encompasses the evaluation of a wide spectrum of lesions. While ultrasound is able to show intra- and peri-articular signs of inflammation and to differentiate active from inactive synovitis with the use of Doppler modes, MR shows also bone marrow inflammatory-related abnormalities. Moreover, ultrasound detects inflammation at multi-site level and allows the measurement of stable remission over time on longitudinal evaluation. Interestingly, also tenosynovial Doppler inflammation has been demonstrated to be a frequent finding in patients in clinical remission and, compared to joint synovitis, it is more associated with self-perceived unstable remission (5). In addition, tenosynovial and joint Doppler positivity has been demonstrated to be an independent risk factor of flare in RA patients in remission (6). Residual joint inflammation in patients in remission can be demonstrated also by other imaging techniques, such as PET which shows enhanced tracer uptake at different joint sites (3). Basically, the capability of imaging to demonstrate sub-clinical disease activity even in patients in clinical remission, by allowing the detection of residual inflammation at different joint sites and at different disease stages gives it an added value compared to clinical assessment alone.

In conclusions, clinical evaluation of remission alone appears frequently inadequate to capture a real persistent lack of inflammation at different joint sites Imaging can complement the assessment of patients in clinical remission, offering additional data that may still reveal a certain amount of sub-clinical disease activ ity. With new therapeutic strategies RA management has become increasingly complex over the last years (7). The relevance of this information is then substantial, being structural damage the result of both previous and current phases of dis ease activity.

\section{REFERENCES:}

[1] Felson, et al. Ann Rheum Dis 2011;70: 404-13.

[2] Chandrashekara, et al. International Journal of Rheumatic Diseases 2013; 16: 291-6.

[3] Bugatti, et al. Front Med (Lausanne)2018; 15: 140-8.

[4] Pope, et al. Athritis Care Res (Hoboken) 2019 Mar 1. doi: 10.1002/ acr.23862.

[5] Bellis, et al. Rheumatology 2016; 55: 1826-36.

[6] Filippou, et al. Ann Rheum Dis 2018; 77: 1283-9.

[7] Smolen, et al. Ann Rheum Dis 2017; 76: 960-77.

Disclosure of Interests: None declared

DOI: 10.1136/annrheumdis-2019-eular.8504

\section{\begin{tabular}{|l|l}
\hline SP0125 HOW TO ASSESS PATIENT WITH SUSPECTED \\
\hline
\end{tabular} VASCULITIS}

Raashid Luqmani. University of Oxford, Nuffield Department of Orthopaedics, Rheumatology and Musculoskeletal Sciences, Oxford, United Kingdom

Background: Systemic vasculitides are multi-organ, multi-system conditions which can mimic many other diseases. They are rare but very significant because they can rapidly lead to end organ damage or death if left untreated. The difficulty faced by clinicians in diagnosing a patient with vasculitis is further compounded by the presentation of limited forms of vasculitis, which can be difficult to recognise as due to vasculitis, the effects of co-existing co-morbidity and the use of drugs either for medicinal or recreational purposes. Giant cell arteritis is one of the most common serious forms of vasculitis in adults, leading to blindness if untreated. In view of this risk, many clinicians will initiate treatment of any patients with sus pected giant cell arteritis before definitive investigations have been performed. Unfortunately this can result in inadequate outcomes form diagnostic tests such as ultrasound or biopsy of the temporal artery. There are no diagnostic criteria for most forms of vasculitis apart from Behcet's syndrome. Some tests can be very useful in ruling out other causes as well as helping to confirm a diagnosis of vasculitis. We will review some of the strategies for diagnosing vasculitis, complementing a thorough history and examination with selected investigations to improve diagnostic certainty.

Objectives: To consider the strategies used to assist the diagnosis of systemic vasculitis; to assess the frequency of multi-system involvement in systemic vasculitis; to critically evaluate the role of diagnostic testing in systemic vasculitis.

Methods: A review of current literature on the approach to diagnosis and classification of vasculitis.

Results: Table 1 summarises the clinical manifestations of some forms of primary systemic vasculitis, comparing the different frequencies of the common presenting features. Patients with large vessel vasculitis are usually more distinct as a clinical group from other forms of vasculitis but increasingly we see an overlap between GCA and older patients (especially males) with Takayasu arteritis. There is considerable overlap of clinical features amongst the small vessel vasculitides, despite different immuno-pathogenetic pathways. Amongst the anti-neutrophil cytoplasm antibody (ANCA) associated vasculitides (AAV), the presence of active nephritis characterises most patients with MPA; it is less common in GPA and least common in EGPA. Lung involvement is prevalent in all three forms of AAV but has different characteristics: bronchial wall inflammation with ulceration plus nodules and infiltrates in GPA; transient infiltrates and bronchospasm in EGPA; lung haemorrhage at presentation in MPA, with subsequent risk of developing lung fibrosis. The different patterns of disease activity help in differentiating 